For an employee to be exempt from contributing to the State reserve scheme, it will be necessary for his occupational scheme to provide: a pension equal to $1 \%$ of P.A.Y.E. earnings (subject to an upper limit of $1 \frac{1}{2}$ times the current national average); a widow's pension payable on death before or after retirement equal to $0.5 \%$ of such earnings and, where only the minimum benefits are provided, increases in the pension to maintain its real value. As the N.H.S. scheme already meets the first two provisions it should be an exempt scheme and its members would not contribute to the State scheme. These particular proposals, therefore, are unlikely to affect doctors' N.H.S. pensions.

The White Paper, however, also proposed that any benefits which have accrued to a member of any approved occupational pension scheme (and not only one which exempts its members from contributing to the earningsrelated scheme) will have to be preserved in full for any member who leaves service after attaining the age of 26 and completing five years' service. Furthermore, a member leaving in these circumstances could not take a refund of his or her contributions to the scheme. Thus any doctor leaving the N.H.S. after completing five years' service after 1975 would automatically be granted a deferred pension to commence at his normal retirement age, based on the actual service which he had completed at that time.

Nevertheless, a deferred pension granted to a doctor leaving the N.H.S. at, say, age 35 which is fixed in money terms may prove to be of little real value when he eventually receives it 30 years later. Preservation of pension benefits is only likely to be of significant value to the recipient if the deferred pensions granted are increased regularly to offset inflation. The Government, though, has indicated that it intends to review practice on preservation in public sector pension schemes before the new scheme in 1975, and the B.M.A. will obviously have an interest in this review. All this, however, will do nothing to meet the fundamental deficiences of the N.H.S. scheme so far as doctors are concerned.

The Secretary of State's letter rejecting the B.M.A.'s proposals for an independent review of pensions and for a separate funded scheme gave as the main reason that the Government sees doctors and dentists broadly as no different from other employees in the public sector. Many public sector employees, however, are members of funded pension schemes, for example the nationalized industries, the Post Office, and local government. Furthermore, the police and the armed Forces already enjoy specially designed benefits.

The pensions of N.H.S. general practitioners are based on earnings throughout their careers so that inflation is particularly serious for them. Earnings of many family doctors tend to fall during the last few years before retirement, and the usual type of calculation which relates pensions to final earnings - as is the case with hospital doctors-has not been regarded as appropriate. But the present method means that in a period of rapid pay rises a retiring general practitioner could well be up to $50 \%$ better off if his pension was calculated on the last few years of earning.

Sir Keith Joseph had promised to look at this point when the Government Actuary produces his septennial report on the scheme-supposedly about mid-1972. But the G.M.S. Committee, however, saw the situation as so urgent that it asked to meet Department of Health officials as soon as possible. The Council at its December meeting had already condemned Sir Keith Joseph's reply as unacceptable to doctors as a whole, referring to their special problems such as late entry, the total earnings basis for G.P.'s pensions, and the pension abatement rules. It also suggested that an ad hoc working party should be set up with representatives from the profession and department to study the B.M.A.'s complaints.

A further letter on superannuation from Sir Keith Joseph -more conciliatory in tone than his earlier one-was discussed by the Council at its January meeting. It referred to proposals, now being studied by the N.H.S. Joint Superannuation Committee, for improving the N.H.S. scheme as a whole, which the Secretary of State thinks may help the profession's position. In the meantime the particular problems of general practitioners as independent contractorsacknowledged by Sir Keith-are being discussed directly with the Department of Health. The whole profession will certainly expect these developments to augur a prompt and equitable outcome to the B.M.A.'s submissions, for inflation continues apace and doctors are increasingly restive about their present pension prospects.

1 Strategy for Pensions (The Future Development of State and Occupational Provision), Cmnd. 4755. London H.M.S.O., 1971.

\section{Pancreatic Transplantation}

Diabetes mellitus is such a common disease that it is not surprising that for some years surgeons have been interested in the possibility of transplanting the pancreas in order to control carbohydrate metabolism. The first operation in man was in 1966 by W. D. Kelly and colleagues. ${ }^{1}$ At least 24 human pancreatic grafts have now been carried out but the indications for the procedure are still far from agreed.

The complications of immunosuppresive therapy, the surveillance of patients, the administration of immunosuppressive drugs, and the likelihood of rejection together act as major deterrents; indeed otherwise pancreatic transplantation would have been widely practised as the surgery is fairly straightforward. But in practice regular administration of insulin or other drugs for the control of diabetes is considerably simpler in most cases than pancreatic grafting.

There are, however, patients whose diabetes is difficult to control with routine therapy and there is an important group of patients who develop serious and progressive diabetic angiopathy, which leads to blindness, renal failure, and neuropathy. In such cases, where the prognosis is poor, it seems justifiable to investigate the possibility of a pancreatic graft not only to substitute for insulin but possibly through some other unknown actions to control the angiopathy. There is, however, no clear evidence that progressive angiopathy is due to an absence of pancreatic function, and pancreatic grafting has not so far been shown to prevent angiopathy from progressing, though a transplanted pancreas can certainly produce good control of carbohydrate metabolism.

Patients with kidney grafts often require large doses of steroids, which can induce a diabetic state in an otherwise previously normal person; and established diabetes can be aggravated by steroid treatment. Diabetic nephropathy is not, therefore, usually considered a good indication for renal grafting and many patients with this condition have failed to be selected for transplant programmes as a consequence. But since renal failure due to diabetic nephropathy is a common complication of severe progressive diabetes, transplanting both kidney and pancreas in such patients seems a possible solution to their difficulties. 
In 197123 pancreatic transplants were reported to the Transplant Registry. ${ }^{2}$ Fifteen patients received kidney transplants as well as pancreatic grafts. Two grafts were in the neck, the remainder in the iliac fossa. Seventeen patients were given pancreaticoduodenal grafts. The graft is vascularized by anastomosing a portion of the aorta containing the coeliac and superior mesenteric arterial orifices to a systemic artery in the recipient, usually one of the iliac vessels, and a portal vein is then anastomosed to an iliac vein. The venous return from the pancreas therefore bypasses the liver, and this may be important in relation to the action of insulin secreted by the pancreas and unmodified by first contact with the patient's liver cells. Immunosuppression has been the same as when used for kidney grafts.

In seven patients graft function continued for three months or more. There was satisfactory control of the blood glucose in most of the patients without the need for any other antidiabetic drugs. None of the patients is now alive. There have been complications in the duodenal portion of the graft and ulceration and perforation. Fifteen patients survived less than three months, with complications of sepsis and technical failure. Where the kidney and pancreas have been transplanted from the same donor evidence of rejection has been more severe in the kidney than in the pancreatic graft. The longest survivor died one year after grafting, having then a rejected kidney but a functioning pancreas.

On the whole, then, the results of pancreatic transplantation seem disappointing. Survival has not been long enough to provide evidence to show whether control of carbohydrate metabolism by a pancreatic graft will prevent progressive angiopathy or whether the alternative theory is correct, that the angiopathy is primary and the abnormality of carbohydrate metabolism a consequence of it. With this background there is unlikely to be widespread clinical application of pancreatic transplantation. For the young patient, however, with severe diabetes that is difficult to control, who is dying of renal failure, and for whom no other therapy is available, combined kidney and pancreatic allografting seems worthy of further trial-if the patient is otherwise unsuitable for management by recurrent dialysis or renal transplantation alone.

\footnotetext{
1 Kellv. W. D.. et al., Surgery, 1971, 61, 827.

2 fournal of the American Medical Association, 1971, 217, 1520.
}

\section{Research in Diseases of the Tropics}

Undoubtedly at present in Britain there is a wealth of interesting and important research being carried out into diseases of the tropics. This is the inescapable reaction to reading the recent issue of the British Medical Bulletin, ${ }^{1}$ in which 16 articles are presented by authorities in the different fields.

R. J. W. Rees and M. F. R. Waters present evidence that in experimental infection with Mycobacterium leprae multiplication is dependent on the host rather than on the strain or virulence of the organism. Establishment of infection in the mouse, in which the pathological changes are almost identical with those found in man, has enabled drugs to be tested. Thus these workers were able to show the rapid bactericidal action of rifampicin and to predict the value of much smaller doses of sulphone than had previously been employed in man. Human lymph node studies have supported a basic immunological difference between lepromatous and tuberculoid leprosy: in lepromatous patients the evidence favours a failure of specific cell-mediated immunity but with the stimulation of humoral antibody production; in those with the tuberculoid form of leprosy the reverse is found. Immunofluorescent techniques have shown that erythema nodosum leprosum is an immune complex disease, whereas lepra reactions are accompanied by a change in cell-mediated immunity. Though the clinical response to low doses of sulphones has been good, it is not yet known whether this will be followed by a lower incidence of erythema nodosum leprosum or a greater incidence of resistance.

Another finding in the mouse was the presence of organisms in striated muscle, and this has now also been shown to be true for man even when organisms are absent in the skin of treated patients. This suggests that in future biopsy of striated muscle will be an important investigation in patients under treatment. The value of B.C.G. for immunization against leprosy seems to vary; whereas in Uganda and New Guinea it did appear to protect children immunized, in Burma this effect was not found. The reason for this difference is not known.

Cell-mediated immunity has also been found to be of importance in schistosomiasis. S. R. Smithers suggests that the granulomatous reaction around the ova in the tissues is a delayed hypersensitivty phenomenon produced specifically by an antigen which has already been partially purified and which is derived only from eggs. Using the rhesus monkeySchistosoma mansoni system, he has found that the living adult worm is the stimulus to immunity. Challenge with cercariae is completely resisted, though the worms constituting the primary infection continue to produce eggs. An explanation of how the adult worm circumvents the host's response (which it has itself induced) is that the adult schistosome has host antigen on its surface; whether this is acquired from the host or manufactured by the parasite is not yet known.

Tropical sprue-that curious condition which is still so poorly understood-is considered by S. J. Baker. Whether the spectrum of disease from fully developed sprue to persons showing mild changes on biopsy of the small bowel but with no demonstrable malabsorption is a single entity is not known. Though no infective agent has been constantly associated, indirect evidence suggests that one or more may play a part. The occurrence of epidemics of sprue-and especially the concentration of cases in young children when a second epidemic follows a first after some years-together with the favourable response that may follow the administration of a tetracycline are consistent with an infective aetiology. Steatorrhoea, which is a regular feature of sprue, seems to be due to various factors-including abnormalities of the bile salts, mucosal cell damage, and abnormal fat transport from the cells to the lymphatics. The finding in $14 \%$ of patients with sprue that intrinsic factor output is in the range seen in pernicious anaemia suggests that anatomical changes are widespread.

Though three articles have been chosen for special comment, all the remainder give authoritative accounts of work being carried out in various aspects; and the whole issue of this British Medical Bulletin will repay study by those working outside the specialty of tropical medicine as well as by those more closely concerned.

\footnotetext{
1 British Medical Bulletin, 1972, 28, No. 1.
} 